\title{
A Hopf-Lax formula for Hamilton-Jacobi equations with Caputo time-fractional derivative
}

\author{
Fabio Camilli* \\ Raul De Maio* \\ Elisa Iacomini*
}

June 4, 2019

\begin{abstract}
We prove a representation formula of Hopf-Lax type for solutions to Hamilton-Jacobi equation involving a Caputo time-fractional derivative. Equations of this type are associated with optimal control problems where the controlled dynamics is given by a time-changed stochastic process describing the trajectory of a particle subject to random trapping effects.
\end{abstract}

AMS subject classification: 35R11, 26A33, 49L20.

Keywords: Caputo derivative, Riemann-Liouville derivative, fractional Hamilton-Jacobi equation, Hopf-Lax formula, subordinator.

\section{Introduction}

In the recent times, several classical parabolic equations have been revisited by replacing the standard time derivative with a fractional one $[1,3,5,9,11,17]$. Time-fractional derivatives are given by convolution integrals of the time derivative with power-law kernels. They arise in several phenomena in connection with anomalous diffusion and are typical for memory effects in complex systems (see [15] for a review). The probabilistic interpretation of the corresponding physical models leads to the study of subdiffusive or, more in general, nonmarkovian processes. From a mathematical point of view, the presence of nonlocal terms with respect to the time variable poses several technical difficulties.

Aim of this paper is to study the connection between Hamilton-Jacobi equations and subdiffusions, recovering a subordinated version of the Hopf-Lax formula. Consider the Cauchy problem

$$
\begin{cases}\partial_{t} u+H(D u)=0 & (x, t) \in Q, \\ u(x, 0)=g(x) & x \in \mathbb{R}^{d},\end{cases}
$$

where $Q=\mathbb{R}^{d} \times(0, \infty)$. In all the paper, we assume that

$$
\begin{gathered}
H: \mathbb{R}^{d} \rightarrow \mathbb{R} \text { is convex and } \lim _{|p| \rightarrow \infty} \frac{H(p)}{|p|}=+\infty ; \\
g: \mathbb{R}^{d} \rightarrow \mathbb{R} \text { is Lipschitz continuous and bounded. }
\end{gathered}
$$

\footnotetext{
${ }^{1}$ Dip. di Scienze di Base e Applicate per l'Ingegneria, "Sapienza" Università di Roma, via Scarpa 16, 00161 Roma, Italy, (e-mail: fabio.camilli,raul.demaio,elisa.iacomini@sbai.uniroma1.it)
} 
Observe that, by (1.2), The Legendre transform $L$ of $H$, defined by $L(q)=\sup \{p q-H(p)\}$, is well defined, convex and superlinear. Then, the classical Hopf-Lax formula

$$
u(x, t)=\min _{y \in \mathbb{R}^{d}}\left\{t L\left(\frac{x-y}{t}\right)+g(y)\right\},
$$

where $L$ is the Legendre transform of $H$, gives the unique viscosity solution of (1.1). Moreover, $u$ is a maximum element of the set of the almost everywhere (a.e.) subsolutions of (1.1) ([8, Theorem 11.1]). Formula (1.4) is derived from the optimal control interpretation of the Cauchy problem. Indeed, the Hamilton-Jacobi equation in (1.1) can be interpreted as the dynamic programming equation satisfied by the value function

$$
u(x, t)=\inf \left\{\int_{0}^{t} L(\dot{Y}(s)) d s+g(Y(0)): Y(t)=x\right\},
$$

with the infimum taken over all the $C^{1}$ functions $Y$ that satisfy the constraint $Y(t)=x$. The velocity $a=\dot{Y}$ can be interpreted as a control variable for the problem. Since $L$ is independent of $(x, t)$, straight lines are proved to be the minimizing trajectories in (1.5) and (1.4) is so obtained (see [4, 8] for details). In this paper we consider the Cauchy problem

$$
\begin{cases}\partial_{(0, t]}^{\beta} u+H(D u)=0 & (x, t) \in Q, \\ u(x, 0)=g(x) & x \in \mathbb{R}^{d},\end{cases}
$$

where

$$
\partial_{(0, t]}^{\beta} u(x, t)=\frac{1}{\Gamma(1-\beta)} \int_{0}^{t} \frac{\partial_{\tau} u(x, \tau)}{(t-\tau)^{\beta}} d \tau
$$

is the Caputo time-fractional derivative of order $\beta \in(0,1)$ of $u$. To deduce a Hopf-Lax formula for (1.6) we rely, as in the classical case, on the optimal control interpretation of the problem. Let $E_{t}$ be a continuous, nondecreasing stochastic process defined as the inverse of a $\beta$-stable subordinator $D_{t}$, i.e. $E_{t}:=\inf \{\tau>0$ : $\left.D_{\tau}>t\right\}$ for $t \geq 0$. The process $X(s)=Y\left(E_{s}\right)$ solves the stochastic differential equation

$$
\left\{\begin{array}{l}
d X(s)=\alpha(s) d E_{s}, \\
X(0)=\xi
\end{array}\right.
$$

where $\alpha(s)=a\left(E_{s}\right)$ and $\xi=Y(0)$. The inverse subordinator $E_{t}$ can be interpreted as a change of the time scale which introduces trapping events in the evolution of the process $X(s)$, whereas, when not trapped, the particle moves according to the standard dynamics $Y(s)$. Consider the value function

$$
u_{\beta}(x, t)=\inf _{\alpha \in \mathcal{A}}\left\{\mathbb{E}_{x, t}\left[\int_{0}^{t} L(\alpha(s)) d E_{s}+g(X(0))\right]: X(t)=x\right\}
$$

where $X$ is a solution of (1.7) and $\alpha$ is the control variable in the class $\mathcal{A}:=\left\{\alpha=\{\alpha(s)\}_{s \geq 0}: \alpha\right.$ is a locally bounded, progressively of the control processes. It is clear that straight lines are still the optimal trajectories minimizing (1.8), but travelled at a velocity which depends on the time scale $E_{t}$. We prove that the value function $u_{\beta}$ associated to the time-changed control problem is given by the Hopf-Lax formula

$$
u_{\beta}(x, t)=\mathbb{E}_{x, t}\left[\min _{y \in \mathbb{R}^{d}}\left\{E_{t} L\left(\frac{x-y}{E_{t}}\right)+g(y)\right\}\right] .
$$

The previous formula is similar to (1.4), but it takes into account the average speed at which the straight trajectories are traveled. We also prove that, as in the classical case, the value function $u_{\beta}$ is a maximum 
element in the set of the a.e. subsolutions of problem (1.6) and also an a.e. solution, but we are not able to prove that it is a viscosity solution in the sense of the definition introduced in [5, 17] (see Remark 3.4 for more details).

We can rewrite formula (1.9) as the convolution of the solution of (1.1) with a kernel $\mathcal{E}_{\beta}$ given by the probability density function $(\mathrm{PDF})$ of the process $E_{t}$, i.e.

$$
u_{\beta}(x, t)=\int_{0}^{\infty} u(x, s) \mathcal{E}_{\beta}(s, t) d s .
$$

Employing a standard numerical solver for (1.1) to compute $u$, we use the previous formula to illustrate with a numerical example the effect of the Caputo time-fractional derivative on the Hamilton-Jacobi equation.

The paper is organized as follows. In Section 2, we briefly recall some properties of the subordinator process and we introduce the Hopf-Lax formula. Section 3 is devoted to the time-fractional Hamilton-Jacobi equation. Finally, in Section 4, a numerical example is discussed in order to stress the main differences with the classical theory.

\section{The subordinator process and the Hopf-Lax formula}

Fixed a filtered probability space $\left(\Omega, \mathcal{F}, \mathcal{F}_{t}, \mathbb{P}\right)$, let $\left\{D_{\tau}\right\}_{\tau \geq 0}$ be a stable subordinator of order $\beta \in(0,1)$, i.e. a one-dimensional, nondecreasing Lévy process whose probability density function (PDF) has Laplace transform

equal to $e^{-\tau s^{\beta}}$. The inverse stable process $\left\{E_{t}\right\}_{t \geq 0}$, defined as the first passage time of the process $D_{\tau}$ over the level $t$, i.e.

$$
E_{t}=\inf \left\{\tau>0: D_{\tau}>t\right\}
$$

has sample paths which are continuous, nondecreasing and such that $E_{0}=0, E_{t} \rightarrow \infty$ as $t \rightarrow \infty$. It is worthwhile to observe that $E_{t}$ does not have stationary or independent increments. The process $E_{t}$ can be used to model systems with two time scales: a deterministic one given by the standard time $t$, referred to an external observer, and a stochastic one given by $E_{t}$, internal to the physical process (see $[10,13,14]$ ).

We recall some basic properties of the process $E_{t}$ which we will exploit in the following.

Proposition 2.1. For $t>0$, it holds:

- For any $\lambda>0$, there exists a constant $C(\lambda, \beta)>0$ such that

$$
\mathbb{E}\left[E_{t}^{\lambda}\right]=C(\lambda, \beta) t^{\lambda \beta} .
$$

with $C(\lambda, \beta)=\frac{\Gamma(\lambda+1)}{\Gamma(\lambda \beta+1)}$.

- The process $E_{t}$ has PDF

$$
\mathcal{E}_{\beta}(s, t)=\frac{t}{\beta} s^{-1-\frac{1}{\beta}} \mathcal{D}_{\beta}\left(t s^{-1 / \beta}\right)
$$

where $\mathcal{D}_{\beta}$ is the PDF of $D_{1}$.

For the proof of the following result we refer to [14].

Proposition 2.2. The function $\mathcal{E}_{\beta}(\cdot, t)$ is a weak solution, in sense of distributions, of

$$
\partial_{(0, t]}^{\beta} \mathcal{E}_{\beta}(r, t)=-\partial_{r} \mathcal{E}_{\beta}(r, t)-\frac{t^{\beta}}{\Gamma(1-\beta)} \delta_{0}(r), \quad r \in(0, \infty) .
$$


Proposition 2.3. The value function $u_{\beta}$ defined in (1.8) is given by the Hopf-Lax formula

$$
u_{\beta}(x, t)=\mathbb{E}_{x, t}\left[\min _{y \in \mathbb{R}^{d}}\left\{E_{t} L\left(\frac{x-y}{E_{t}}\right)+g(y)\right\}\right] .
$$

Proof. Fix $(x, t) \in \mathbb{R}^{d} \times(0,+\infty)$. Let $Y$ be a random variable such that

$$
\min _{y \in \mathbb{R}^{d}}\left\{E_{t} L\left(\frac{x-y}{E_{t}}\right)+g(y)\right\}=E_{t} L\left(\frac{x-Y}{E_{t}}\right)+g(Y) .
$$

Note that $Y$ is well defined since, by (1.2) and (1.3), the minimum in the left-hand side of (2.5) is achieved for any $\omega \in \Omega$. For the control law $\bar{a}(s)=(x-Y) / E_{t}$, consider the solution $X(s)$ of $(1.7)$. Then

$$
X(s)=Y+\frac{x-Y}{E_{t}} E_{s}
$$

and therefore $X(0)=Y$ (recall that $E_{0}=0$ ) and $X(t)=x$. Hence

$$
\begin{aligned}
u_{\beta}(x, t) & \leq \mathbb{E}_{x, t}\left\{\int_{0}^{t} L\left(\frac{x-Y}{E_{t}}\right) d E_{s}+g(X(0))\right\}=\mathbb{E}_{x, t}\left\{E_{t} L\left(\frac{x-Y}{E_{t}}\right)+g(Y)\right\} \\
& =\mathbb{E}_{x, t}\left[\min _{y \in \mathbb{R}^{d}}\left\{E_{t} L\left(\frac{x-y}{E_{t}}\right)+g(y)\right\}\right] .
\end{aligned}
$$

We prove the reverse inequality. Given a control $\alpha \in \mathcal{A}$, by the convexity of $L$ we have

$$
L\left(\frac{1}{E_{t}} \int_{0}^{t} \alpha(s) d E_{s}\right) \leq \frac{1}{E_{t}} \int_{0}^{t} L(\alpha(s)) d E_{s}
$$

If $X(t)$ is the solution of (1.7), since $\int_{0}^{t} \alpha(s) d E_{s}=x-X(0)$, by the previous inequality we get

$$
\begin{aligned}
\mathbb{E}_{x, t}\left\{\int_{0}^{t} L(\alpha(s)) d E_{s}+g(X(0))\right\} & \geq \mathbb{E}_{x, t}\left\{E_{t} L\left(\frac{x-X(0)}{E_{t}}\right)+g(X(0))\right\} \\
& \geq \mathbb{E}_{x, t}\left[\min _{y \in \mathbb{R}^{d}}\left\{E_{t} L\left(\frac{x-y}{E_{t}}\right)+g(y)\right\}\right]
\end{aligned}
$$

and, for the arbitrariness of $a \in \mathcal{A}$,

$$
u_{\beta}(x, t) \geq \mathbb{E}_{x, t}\left[\min _{y \in \mathbb{R}^{d}}\left\{E_{t} L\left(\frac{x-y}{E_{t}}\right)+g(y)\right\}\right] .
$$

In order to prove some regularity properties of the function $u_{\beta}$, we need a preliminary result.

Lemma 2.4. For $s \in[0, t)$, we have

$$
u_{\beta}(x, t)=\mathbb{E}_{x, t}\left[\min _{y \in \mathbb{R}^{d}}\left\{\left(E_{t}-E_{s}\right) L\left(\frac{x-y}{E_{t}-E_{s}}\right)+u_{\beta}(y, s)\right\}\right] .
$$

Proof. For $y \in \mathbb{R}^{d}$, let $Z: \Omega \rightarrow \mathbb{R}^{d}$ be a random variable such that

$$
u_{\beta}(y, s)=\mathbb{E}_{x, t}\left[E_{s} L\left(\frac{y-Z}{E_{s}}\right)+g(Z)\right] .
$$

By the identity

$$
\frac{x-Z}{E_{t}}=\left(1-\frac{E_{s}}{E_{t}}\right) \frac{x-y}{E_{t}-E_{s}}+\frac{E_{s}}{E_{t}} \frac{y-Z}{E_{s}}
$$


and by the convexity of $L$, we get

$$
L\left(\frac{x-Z}{E_{t}}\right) \leq\left(1-\frac{E_{s}}{E_{t}}\right) L\left(\frac{x-y}{E_{t}-E_{s}}\right)+\frac{E_{s}}{E_{t}} L\left(\frac{y-Z}{E_{s}}\right) .
$$

Therefore

$$
\begin{aligned}
u_{\beta}(x, t) & =\mathbb{E}_{x, t}\left[\min _{y \in \mathbb{R}^{d}}\left\{E_{t} L\left(\frac{x-y}{E_{t}}\right)+g(y)\right\}\right] \leq \mathbb{E}_{x, t}\left[E_{t} L\left(\frac{x-Z}{E_{t}}\right)+g(Z)\right] \\
& \leq \mathbb{E}_{x, t}\left[\left(E_{t}-E_{s}\right) L\left(\frac{x-y}{E_{t}-E_{s}}\right)+E_{s} L\left(\frac{y-Z}{E_{s}}\right)+g(Z)\right] \\
& =\mathbb{E}_{x, t}\left[\left(E_{t}-E_{s}\right) L\left(\frac{x-y}{E_{t}-E_{s}}\right)+u_{\beta}(y, s)\right] .
\end{aligned}
$$

Since the previous inequality holds for any $y \in \mathbb{R}^{d}$, we get

$$
u_{\beta}(x, t) \leq \mathbb{E}_{x, t}\left[\min _{y \in \mathbb{R}^{d}}\left\{\left(E_{t}-E_{s}\right) L\left(\frac{x-y}{E_{t}-E_{s}}\right)+u_{\beta}(y, s)\right\}\right] .
$$

To prove the reverse inequality, let $W$ be a random variable such that

$$
u_{\beta}(x, t)=\mathbb{E}_{x, t}\left[\min _{y \in \mathbb{R}^{d}}\left\{E_{t} L\left(\frac{x-y}{E_{t}}\right)+g(y)\right\}\right]=\mathbb{E}_{x, t}\left[E_{t} L\left(\frac{x-W}{E_{t}}\right)+g(W)\right] .
$$

If $Y: \Omega \rightarrow \mathbb{R}^{d}$ is a random variable, since

$$
\mathbb{E}_{x, t}\left[u_{\beta}(Y, s)\right] \leq \mathbb{E}_{x, t}\left[E_{s} L\left(\frac{Y-W}{E_{s}}\right)+g(W)\right],
$$

it follows that

$$
u_{\beta}(x, t) \geq \mathbb{E}_{x, t}\left[E_{t} L\left(\frac{x-W}{E_{t}}\right)-E_{s} L\left(\frac{Y-W}{E_{s}}\right)+u_{\beta}(Y, s)\right] .
$$

Set $Y=\frac{E_{s}}{E_{t}} x+\left(1-\frac{E_{s}}{E_{t}}\right) W$. Then $\frac{x-Y}{E_{t}-E_{s}}=\frac{x-W}{E_{t}}=\frac{Y-W}{E_{s}}$ and by $(2.7)$

$$
\begin{aligned}
u_{\beta}(x, t) & \geq \mathbb{E}_{x, t}\left[\left(E_{t}-E_{s}\right) L\left(\frac{x-Y}{E_{t}-E_{s}}\right)+u_{\beta}(Y, s)\right] \\
& \geq \mathbb{E}_{x, t}\left[\min _{y \in \mathbb{R}^{d}}\left\{\left(E_{t}-E_{s}\right) L\left(\frac{x-y}{E_{t}-E_{s}}\right)+u_{\beta}(y, s)\right\}\right] .
\end{aligned}
$$

Remark 2.5. Arguing as in Lemma 2.4, it is also possible to prove that if $\tau: \Omega \rightarrow[0, t)$ is a stopping time, then

$$
u_{\beta}(x, t)=\mathbb{E}_{x, t}\left[\min _{y \in \mathbb{R}^{d}}\left\{\left(E_{t}-E_{\tau}\right) L\left(\frac{x-y}{E_{t}-E_{\tau}}\right)+u_{\beta}(y, \tau)\right\}\right] .
$$

Proposition 2.6. We have

(i) For all $x, \bar{x} \in \mathbb{R}^{d}, t \in(0, \infty)$

$$
\left|u_{\beta}(x, t)-u_{\beta}(\bar{x}, t)\right| \leq L_{g}|x-\bar{x}|,
$$

where $L_{g}$ is the Lipschitz constant of the initial datum $g$.

(ii) There exists a constant $C$ such that for all $x \in \mathbb{R}^{d}, t \in(0, \infty)$

$$
\left|u_{\beta}(x, t)-g(x)\right| \leq C t^{\beta} .
$$


(iii) There exists a constant $C$ such that for all $x \in \mathbb{R}^{d}, t, \bar{t} \in(0, \infty), \bar{t}<t$,

$$
\left|u_{\beta}(x, t)-u_{\beta}(x, \bar{t})\right| \leq C(t-\bar{t})^{\beta} .
$$

Proof. Fixed $t>0, x, \bar{x} \in \mathbb{R}^{d}$, let $Z: \Omega \rightarrow \mathbb{R}^{d}$ be a random variable such that

$$
E_{t} L\left(\frac{x-Z}{E_{t}}\right)+g(Z)=\min _{y \in \mathbb{R}^{d}}\left\{E_{t} L\left(\frac{x-y}{E_{t}}\right)+g(y)\right\} .
$$

Then

$$
\begin{aligned}
u_{\beta}(\bar{x}, t)-u_{\beta}(x, t) & =\mathbb{E}_{x, t}\left[\min _{y \in \mathbb{R}^{d}}\left\{E_{t} L\left(\frac{\bar{x}-y}{E_{t}}\right)+g(y)\right\}\right]-\mathbb{E}_{x, t}\left[E_{t} L\left(\frac{x-Z}{E_{t}}\right)+g(Z)\right] \\
& \leq \mathbb{E}_{x, t}\left[E_{t} L\left(\frac{x-Z}{E_{t}}\right)+g(\bar{x}-x+Z)\right]-\mathbb{E}_{x, t}\left[E_{t} L\left(\frac{x-Z}{E_{t}}\right)+g(Z)\right] \\
& \leq \mathbb{E}_{x, t}[g(\bar{x}-x+Z)-g(Z)] \leq L_{g}|\bar{x}-x| .
\end{aligned}
$$

Exchanging the role of $x, \bar{x}$, we get $(2.9)$.

Fix $x \in \mathbb{R}^{d}$ and $t>0$. We recall that for any $t, \gamma>0$, the $\gamma$-moment of $E_{t}$ is given by

$$
\mathbb{E}\left(E_{t}^{\gamma}\right)=C(\beta, \gamma) t^{\beta \gamma},
$$

for some positive constant $C(\beta, \gamma)$ (see (2.1)). Then, setting $y=x$ in the right-hand side of (2.4) and recalling (2.1), we get

$$
u_{\beta}(x, t) \leq \mathbb{E}_{x, t}\left[E_{t} L(0)+g(x)\right]=L(0) \mathbb{E}_{x, t}\left[E_{t}\right]+g(x)=L(0) c(\beta, 1) t^{\beta}+g(x) .
$$

To get the other inequality in (2.10), we observe that

$$
\begin{aligned}
u_{\beta}(x, t) & -g(x)=\mathbb{E}_{x, t}\left[\min _{y \in \mathbb{R}^{d}}\left\{E_{t} L\left(\frac{x-y}{E_{t}}\right)+g(y)-g(x)\right\}\right] \\
\geq & \mathbb{E}_{x, t}\left[\min _{y \in \mathbb{R}^{d}}\left\{E_{t} L\left(\frac{x-y}{E_{t}}\right)-L_{g}|x-y|\right\}\right]=-\mathbb{E}_{x, t}\left[E_{t} \max _{z \in \mathbb{R}^{d}}\left\{-L(z)+L_{g}|z|\right\}\right] \\
& =\mathbb{E}_{x, t}\left[-E_{t} \max _{|w| \leq L_{g}} \max _{z \in \mathbb{R}^{d}}\{|w|-L(w)\}\right]=-\max _{|w| \leq L_{g}}\{H(w)\} \mathbb{E}_{x, t}\left[E_{t}\right] .
\end{aligned}
$$

By (2.1) and (2.13), we get (2.10).

To prove (2.11), fix $x \in \mathbb{R}^{d}$ and $0<\bar{t}<t$. Setting $y=x, s=\bar{t}$ in the right-hand side of (2.6) and recalling (2.1), we get

$$
\begin{aligned}
u_{\beta}(x, t) & \leq \mathbb{E}_{x, t}\left[\left(E_{t}-E_{\bar{t}}\right) L(0)+u_{\beta}(x, \bar{t})\right]=L(0) \mathbb{E}_{x, t}\left[E_{t}-E_{\bar{t}}\right]+u_{\beta}(x, \bar{t}) \\
& =L(0) c(\beta, 1)\left(t^{\beta}-\bar{t}^{\beta}\right)+u_{\beta}(x, \bar{t}) \leq L(0) c(\beta, 1)(t-\bar{t})^{\beta}+u_{\beta}(x, \bar{t}) .
\end{aligned}
$$

On the other side, by (2.6) with $s=\bar{t}$ and (2.9), arguing as in (2.14), we have

$$
\begin{aligned}
u_{\beta}(x, t)-u_{\beta}(x, \bar{t}) & =\mathbb{E}_{x, t}\left[\min _{y \in \mathbb{R}^{d}}\left\{\left(E_{t}-E_{\bar{t}}\right) L\left(\frac{x-y}{E_{t}-E_{\bar{t}}}\right)+u_{\beta}(y, \bar{t})-u_{\beta}(x, \bar{t})\right\}\right] \\
& \geq \mathbb{E}_{x, t}\left[\min _{y \in \mathbb{R}^{d}}\left\{\left(E_{t}-E_{\bar{t}}\right) L\left(\frac{x-y}{E_{t}-E_{\bar{t}}}\right)-L_{g}|x-y|\right\}\right] \\
& =\mathbb{E}_{x, t}\left[-\left(E_{t}-E_{\bar{t}}\right) \max _{|w| \leq L_{g}} \max _{z \in \mathbb{R}^{d}}\{|w|-L(w)\}\right] \\
& =-\max _{|w| \leq L_{g}}\{H(w)\} \mathbb{E}_{x, t}\left[E_{t}-E_{\bar{t}}\right] \geq-\max _{|w| \leq L_{g}}\{H(w)\} c(\beta, 1)(t-\bar{t})^{\beta} .
\end{aligned}
$$




\section{The fractional Hamilton-Jacobi equation}

We exploit the results of the previous section to show that the function $u_{\beta}$ given by (2.4) is an a.e. solution, in the sense given by the next definition, of the Cauchy problem (1.6).

Definition 3.1. A function $v \in C^{0}(\bar{Q})$ is said to be an a.e. subsolution of (1.6) if $D v \in L^{\infty}(Q), \partial_{t} v(x, \cdot) \in$ $L_{l o c}^{1}(0, \infty)$ for all $x \in \mathbb{R}^{d}$ and

$$
\begin{array}{rr}
\partial_{(0, t]}^{\beta} v+H(D v) \leq 0 & \text { a.e. in } Q, \\
v(x, 0) \leq g(x) & x \in \mathbb{R}^{d} .
\end{array}
$$

The function $v$ is said to be an a.e. solution of (1.6) if it attains the equality in (3.1)-(3.2).

Observe that, since $\partial_{t} v(x, \cdot) \in L_{\text {loc }}^{1}(0, T)$, for all $x \in \mathbb{R}^{d}$, then the function $v(x, \cdot)$ is absolutely continuous with respect to $t$ and therefore the time-fractional derivative $\partial_{(0, t]}^{\beta} v(x, t) \in L_{l o c}^{1}(0, T)$ is defined for a.e. $(x, t) \in Q$ (see for example [7, Lemma 2.17]).

Proposition 3.2. The function $u_{\beta}$ is a maximum element in the set of the a.e. subsolutions of (1.6), i.e. $v \leq u_{\beta}$ in $\bar{Q}$ for any a.e. subsolution $v$. In addition, $u_{\beta}$ is an a.e. solution of (1.6).

Proof. By Proposition 2.6, we have that $D u_{\beta} \in L^{\infty}(Q), \partial_{t} u_{\beta}(x, \cdot) \in L_{\text {loc }}^{1}(0, \infty)$ for all $x \in \mathbb{R}^{d}$. Given $(x, t) \in$ $\mathbb{R}^{d} \times(0, \infty)$ such that $D u_{\beta}(x, t)$ exists, we prove that for any $q \in \mathbb{R}^{d}$,

$$
\partial_{(0, t]}^{\beta} u_{\beta}(x, t)+D u_{\beta}(x, t) \cdot q-L(q) \leq 0 .
$$

Indeed, fix $q \in \mathbb{R}^{d}$ and $h>0$. Consider the control law $\alpha(s) \equiv q$. Then the solution $X(s)$ of (1.7) is given by $X(s)=x-\left(E_{t}-E_{s}\right) q$. Define the stopping time

$$
\tau_{h}=\sup \{s \in(t-h, t):|X(s)-x|=h\} .
$$

By (2.8), for $\tau=\tau_{h}$ and $y=X\left(\tau_{h}\right)$, we have

$$
u_{\beta}(x, t) \leq \mathbb{E}_{x, t}\left[\left(E_{t}-E_{\tau_{h}}\right) L\left(\frac{x-X\left(\tau_{h}\right)}{E_{t}-E_{\tau_{h}}}\right)+u_{\beta}\left(X\left(\tau_{h}\right), \tau_{h}\right)\right] .
$$

Since $q=\left(x-X\left(\tau_{h}\right)\right) /\left(E_{t}-E_{\tau_{h}}\right)$,

$$
\mathbb{E}_{x, t}\left[u_{\beta}(X(t), t)-u_{\beta}\left(X\left(\tau_{h}\right), \tau_{h}\right)\right] \leq L(q) \mathbb{E}_{x, t}\left[E_{t}-E_{\tau_{h}}\right] .
$$

By Ito's formula [6], we also have

$$
\begin{aligned}
& \mathbb{E}_{x, t}\left[u_{\beta}(X(t), t)-u_{\beta}\left(X\left(\tau_{h}\right), \tau_{h}\right)\right]=\mathbb{E}_{x, t}\left[\int_{\tau_{h}}^{t} d u_{\beta}(X(s), s)\right] \\
& \mathbb{E}_{x, t}\left[\int_{\tau_{h}}^{t} \partial_{s} u_{\beta}(X(s), s) d s+\int_{\tau_{h}}^{t} D u_{\beta}(X(s), s) d X(s)\right] \\
& =\mathbb{E}_{x, t}\left[\int_{\tau_{h}}^{t} \partial_{s} u_{\beta}(X(s), s) d s+\int_{\tau_{h}}^{t} D u_{\beta}(X(s), s) \cdot q d E_{s}\right] .
\end{aligned}
$$


Therefore, recalling (3.4),

$$
\begin{aligned}
\mathbb{E}_{x, t} & {\left[\int_{\tau_{h}}^{t} \partial_{s} u_{\beta}(X(s), s) d s+\int_{\tau_{h}}^{t}\left(D u_{\beta}(X(s), s) \cdot q-L(q)\right) d E_{s}\right]=\mathbb{E}_{x, t}\left[\int_{\tau_{h}}^{t} \partial_{s} u_{\beta}(X(s), s) d s\right.} \\
& \left.+\int_{0}^{+\infty}\left(\int_{0}^{r}\left(D u_{\beta}\left(Y(s), D_{s}\right) \cdot q-L(q)\right) d s\right)\left(\mathcal{E}_{\beta}(r, t)-\mathcal{E}_{\beta}\left(r, \tau_{h}\right)\right) d r\right] \leq 0
\end{aligned}
$$

where $D_{s}$ is the inverse of $E_{t}$, i.e. $E_{D_{s}}=s$. Dividing the previous inequality by $h$ and passing to the limit for $h \rightarrow 0^{+}$, by the Dominated Convergence Theorem we get

$$
\partial_{t} u_{\beta}(x, t)+\mathbb{E}_{x, t}\left[\int_{0}^{+\infty}\left(\int_{0}^{r}\left(D u_{\beta}\left(Y(s), D_{s}\right) \cdot q-L(q)\right) d s\right) \partial_{t} \mathcal{E}_{\beta}(r, t) d r\right] \leq 0 .
$$

Set $\Phi(r)=\int_{0}^{r}\left(D u_{\beta}\left(Y(s), D_{s}\right) \cdot q-L(q)\right) d s$. Since $\mathcal{E}_{\beta}$ is a solution of $(2.3)$, then we have (see [3, Lemma 4.2])

$$
\partial_{t} \mathcal{E}_{\beta}(r, t)=-D_{(0, t]}^{1-\beta}\left[\partial_{r} \mathcal{E}_{\beta}(r, t)\right]-\delta_{0}(r) \delta_{0}(t) \quad r \in(0, \infty),
$$

where $D_{(0, t]}^{1-\beta}$ is the Riemann-Liouville derivative of order $1-\beta$, which is defined for a continuous function $f:[0, t] \rightarrow \mathbb{R}$ by

$$
D_{(0, t]}^{1-\beta} f(t):=\frac{1}{\Gamma(\beta)} \frac{d}{d t} \int_{0}^{t} f(\tau) \frac{1}{(t-\tau)^{1-\beta}} d \tau
$$

Therefore

$$
\begin{aligned}
\mathbb{E}_{x, t}\left[\int_{0}^{+\infty} \Phi(r) \partial_{t} \mathcal{E}_{\beta}(r, t) d r\right] & =-\mathbb{E}_{x, t}\left[\int_{0}^{+\infty} \Phi(r) D_{(0, t]}^{1-\beta} \partial_{r} \mathcal{E}_{\beta}(r, t) d r\right]-\Phi(0) \delta_{0}(t) \\
& =-\mathbb{E}_{x, t}\left[D_{(0, t]}^{1-\beta}\left(\int_{0}^{+\infty} \Phi(r) \partial_{r} \mathcal{E}_{\beta}(r, t) d r\right)\right] \\
& =-\mathbb{E}_{x, t}\left[D_{(0, t]}^{1-\beta}\left(\left[\Phi(r) \mathcal{E}_{\beta}(r, t)\right]_{0}^{+\infty}-\int_{0}^{+\infty} \partial_{r} \Phi(r) \mathcal{E}_{\beta}(r, t) d r\right)\right] .
\end{aligned}
$$

Since $\lim _{r \rightarrow+\infty} \mathcal{E}_{\beta}(r, t)=0$ and $\partial_{r} \Phi(r)=D u_{\beta}\left(Y(r), D_{r}\right) \cdot q-L(q)$, we have

$$
\begin{aligned}
\mathbb{E}_{x, t}\left[\int_{0}^{+\infty} \Phi(r) \partial_{t} \mathcal{E}_{\beta}(r, t) d r\right] & =\mathbb{E}_{x, t}\left[D_{(0, t]}^{1-\beta}\left(\int_{0}^{+\infty} \mathcal{E}_{\beta}(r, t)\left(D u_{\beta}\left(Y(r), D_{r}\right) \cdot q-L(q)\right) d r\right)\right] \\
& =\mathbb{E}_{x, t}\left[D_{(0, t]}^{1-\beta}\left(D u_{\beta}(X(t), t) \cdot q-L(q)\right)\right] \\
& =D_{(0, t]}^{1-\beta}\left(D u_{\beta}(x, t) \cdot q-L(q)\right) .
\end{aligned}
$$

Replacing (3.8) in (3.6), we get

$$
\partial_{t} u_{\beta}(x, t)+D_{(0, t]}^{1-\beta}\left(D u_{\beta}(x, t) \cdot q-L(q)\right) \leq 0 .
$$

Applying the fractional integral $I_{(0, t]}^{1-\beta} \cdot=\frac{1}{\Gamma(1-\beta)} \int_{0}^{t} \frac{\cdot}{(t-\tau)^{\beta}} d \tau$ to the previous equation, we finally get

$$
\partial_{(0, t]}^{\beta} u_{\beta}(x, t)+D u_{\beta}(x, t) \cdot q-L(q) \leq 0,
$$

hence the claim (3.3). It follows that $u_{\beta}$ is an a.e. subsolution of (1.6).

We now prove that $u_{\beta}$ is a maximum in the set of the subsolutions of the problem. Assume by contradiction that there exist $(x, t) \in Q, \varepsilon>0$ and an a.e. subsolution $v$ of (1.6) such that

$$
u_{\beta}(x, t) \leq v(x, t)-2 \varepsilon .
$$


It is not restrictive to assume that $v \in C^{1}(Q)$ (see Lemma 3.3 at the end of the proof). Let $\alpha$ be an $\varepsilon$-optimal control for $u_{\beta}$, i.e.

$$
u_{\beta}(x, t) \geq \mathbb{E}_{x, t}\left\{\int_{0}^{t} L(\alpha(s)) d E_{s}+g(X(0))\right\}-\varepsilon,
$$

where $X(s)$ is given by the solution of (1.7) corresponding to $\alpha$. By Ito's formula

$$
\begin{aligned}
v(x, t) & =\mathbb{E}_{x, t}[v(X(t), t)] \\
& =\mathbb{E}_{x, t}\left[v(X(0), 0)+\int_{0}^{t} d v(X(s), s)\right] \\
& =\mathbb{E}_{x, t}\left[v(X(0), 0)+\int_{0}^{t} \partial_{s} v(X(s), s) d s+\int_{0}^{t} D v(X(s), s) \cdot \alpha(s) d E_{s}\right] \\
& \leq \mathbb{E}_{x, t}\left[g(X(0))+\int_{0}^{t} \partial_{s} v(X(s), s) d s+\int_{0}^{t}(L(\alpha(s))+H(D v(X(s), s))) d E_{s}\right] \\
& \leq u_{\beta}(x, t)+\varepsilon+\mathbb{E}_{x, t}\left[\int_{0}^{t} \partial_{s} v(X(s), s) d s+\int_{0}^{t} H(D v(X(s), s)) d E_{s}\right] \\
& =u_{\beta}(x, t)+\varepsilon+\mathbb{E}_{x, t}\left[\int_{0}^{t} \partial_{s} v(X(s), s) d s+\int_{0}^{\infty}\left(\int_{0}^{r} H\left(D v\left(Y(s), D_{s}\right)\right) d s\right)\left(\mathcal{E}_{\beta}(r, t)-\mathcal{E}_{\beta}(r, 0)\right) d r\right] \\
& =u_{\beta}(x, t)+\varepsilon+\mathbb{E}_{x, t}\left[\int_{0}^{t} \partial_{s} v(X(s), s) d s+\int_{0}^{\infty} \Phi(r)\left(\mathcal{E}_{\beta}(r, t)-\mathcal{E}_{\beta}(r, 0)\right) d r\right]
\end{aligned}
$$

where $\Phi(r)=\int_{0}^{r} H\left(D v\left(Y(s), D_{s}\right)\right) d s$. Integrating (2.3) and observing that $\mathcal{E}_{\beta}(r, 0)=0$ for all $r \in[0,+\infty)$, we have

$$
\mathcal{E}_{\beta}(r, t)=-I_{(0, t]}^{\beta}\left(\partial_{r} \mathcal{E}_{\beta}(r, \cdot)\right)+\delta_{0}(r) \mathcal{H}(r),
$$

where $\delta_{0}$ and $\mathcal{H}$ are the Dirac function at 0 and the Heaviside function. Performing a computation similar to (3.7), we have that

$$
\begin{aligned}
& \left.\mathbb{E}_{x, t}\left[\int_{0}^{\infty} \Phi(r) \mathcal{E}_{\beta}(r, t)\right) d r\right]=-\mathbb{E}_{x, t}\left[I_{(0, t]}^{\beta}\left(\int_{0}^{\infty} \Phi(r) \partial_{r} \mathcal{E}_{\beta}(r, \cdot)\right) d r\right] \\
& =\mathbb{E}_{x, t}\left[I_{(0, t]}^{\beta}\left(\int_{0}^{\infty} \partial_{r} \Phi(r) \mathcal{E}_{\beta}(r, \cdot)\right) d r\right]=\mathbb{E}_{x, t}\left[I_{(0, t]}^{\beta}\left(\int_{0}^{\infty} H\left(D v\left(Y(r), D_{r}\right)\right) \mathcal{E}_{\beta}(r, \cdot) d r\right)\right] \\
& =\mathbb{E}_{x, t}\left[I_{(0, t]}^{\beta}[H(D v(X(\cdot), \cdot))]\right] .
\end{aligned}
$$

Replacing the previous identity in (3.5), we finally get that

$$
v(x, t) \leq u_{\beta}(x, t)+\varepsilon+\mathbb{E}_{x, t}\left[\int_{0}^{t} \partial_{s} v(X(s), s) d s+I_{(0, t]}^{\beta}[H(D v(X(\cdot), \cdot))]\right] .
$$

Since $v$ is a $C^{1}$ subsolution of (1.6), by applying the operator $I_{(0, t]}^{\beta}[\cdot]$ to the equation satisfied by $v$ we get

$$
\int_{0}^{t} \partial_{s} v(x, s) d s+I_{(0, t]}^{\beta}[H(D v(x, \cdot))] \leq 0 \quad \forall(x, t) \in Q .
$$

Replacing the previous inequality in (3.13), we get a contradiction to (3.11).

We finally prove that $u_{\beta}$ satisfies (1.6) a.e. in $Q$. Assume by contradiction that there exists $\left(x_{0}, t_{0}\right) \in Q$ and $\delta, \varepsilon$ positive such that, defined $U=\left(x_{0}-\delta, x_{0}+\delta\right) \times\left(t_{0}-\varepsilon, t_{0}+\varepsilon\right)$, we have

$$
\partial_{(0, t]}^{\beta} u_{\beta}+H\left(D u_{\beta}\right) \leq-2 \delta<0 \quad \text { a.e. in } U \text {. }
$$


Define the function $\phi(t)=\left(t-\left(t_{0}-\varepsilon\right)\right) \chi_{\left(t_{0}-\varepsilon, t_{0}\right]}+\left(\left(t_{0}+\varepsilon\right)-t\right) \chi_{\left(t_{0}, t_{0}+\varepsilon\right)}$ for $t \in \mathbb{R}$, where $\chi_{[a, b]}$ is the characteristic function of the interval $[a, b]$. Set $C_{\beta}=\varepsilon^{\beta} / \Gamma(2-\beta)$ and observe that $\partial_{(0, t]}^{\beta} \phi(t)=\left(t-t_{0}+\varepsilon\right)^{\beta} / \Gamma(2-\beta)$ for $t \in\left(t_{0}-\varepsilon, t_{0}\right], \partial_{(0, t]}^{\beta} \phi(t)=C_{\beta}-\left(t-t_{0}\right)^{\beta} / \Gamma(2-\beta)$ for $t \in\left(t_{0}, t_{0}+\varepsilon\right)$ and $\partial_{(0, t]}^{\beta} \phi(t)=0$ otherwise. Hence $\partial_{(0, t]}^{\beta} \phi(t) \leq C_{\beta}$ for all $t \in \mathbb{R}$. Defined $\bar{u}(x, t)=u_{\beta}(x, t)+\frac{\delta}{C_{\beta}} \phi(t)$ for $(x, t) \in Q$, by (3.14) we have

$$
\partial_{(0, t]}^{\beta} \bar{u}+H(D \bar{u}) \leq \partial_{(0, t]}^{\beta} u_{\beta}+H\left(D u_{\beta}\right)+\delta \leq-\delta \quad \text { a.e. in } U .
$$

Moreover, if $\varepsilon$ is small enough in such a way that $t_{0}-\varepsilon>0$, it follows that $\bar{u}(x, 0)=u_{\beta}(x, 0)=g(x)$ and therefore $\bar{u}$ is an a.e. subsolution of (1.6). Since $\bar{u}\left(x_{0}, t_{0}\right)=u_{\beta}\left(x_{0}, t_{0}\right)+\frac{\delta}{C_{\beta}}$, we get a contradiction to the maximality of $u_{\beta}$ among the subsolutions of (1.6).

Lemma 3.3. Let $v$ be a subsolution of (1.6). Then there exists a sequence of subsolutions $v_{\delta} \in C^{1}(Q)$ such that $v_{\delta}$ tends to $v$ locally uniformly for $\delta \rightarrow 0$.

Proof. Given a subsolution $v$, we define $v(x, t)=v(x, 0)$ per $t \in(-\infty, 0)$, hence we can write

$$
\partial_{(0, t]}^{\beta} v(x, t)=\frac{1}{\Gamma(1-\beta)} \int_{0}^{t} \frac{\partial_{\tau} v(x, t-\tau)}{\tau^{\beta}} d \tau=\frac{1}{\Gamma(1-\beta)} \int_{0}^{\infty} \frac{\partial_{\tau} v(x, t-\tau)}{\tau^{\beta}} d \tau .
$$

Let $v_{\delta}=v * \rho_{\delta}(x, t)$ where $\rho_{\delta}$ is a standard mollifier in $\mathbb{R}^{d+1}$, i.e. $\rho_{\delta}(\cdot)=\frac{1}{\delta^{d+1}} \rho(\dot{\bar{\delta}})$ with $\rho$ a smooth function such that $\operatorname{supp} \rho \subset\{|x|<1,|t|<1\}$ and $\int_{\mathbb{R}^{d+1}} \rho d x d t=1$. Then $v_{\delta} \rightarrow v$ locally uniformly for $\delta \rightarrow 0$ and by convexity

$$
H\left(D v_{\delta}(x, t)\right) \leq\left(H(D v) * \rho_{\delta}\right)(x, t) \quad \forall(x, t) \in Q .
$$

Moreover

$$
\begin{aligned}
\left(\partial_{(0, t]}^{\beta} v(x, t) * \rho_{\delta}\right)(x, t) & =\int_{\mathbb{R}^{d+1}} \frac{1}{\Gamma(1-\beta)}\left(\int_{0}^{\infty} \frac{\partial_{t} v(y, s-\tau)}{\tau^{\beta}} d \tau\right) \rho_{\delta}(x-y, t-s) d s d y \\
& =\frac{1}{\Gamma(1-\beta)} \int_{0}^{\infty}\left(\int_{\mathbb{R}^{d+1}} \partial_{t} v(y, s-\tau) \rho_{\delta}(x-y, t-s) d s d y\right) \frac{1}{\tau^{\beta}} d \tau \\
& =\frac{1}{\Gamma(1-\beta)} \int_{0}^{\infty}\left(\int_{\mathbb{R}^{d+1}} \partial_{t} v(y, r) \rho_{\delta}(x-y, t-\tau-r) d r d y\right) \frac{1}{\tau^{\beta}} d \tau \\
& =\frac{1}{\Gamma(1-\beta)} \int_{0}^{\infty}\left(\partial_{t} v * \rho_{\delta}\right)(x, t-\tau) \frac{1}{\tau^{\beta}} d \tau=\partial_{(0, t]}^{\beta} v_{\delta}(x, t) .
\end{aligned}
$$

Replacing the previous identity and (3.15) in (3.1), we get

$$
\partial_{(0, t]}^{\beta} v_{\delta}(x, t)+H\left(D v_{\delta}(x, t)\right) \leq 0 \quad \forall(x, t) \in Q .
$$

Since $\left\|v-v_{\delta}\right\|_{\infty} \leq C \delta$, with $C$ depending on $\|D v\|_{\infty}$, by subtracting $C \delta$ to $v_{\delta}$, we have that $v_{\delta}$ also satisfies (3.2).

Remark 3.4. It is well known that Hamilton-Jacobi equations such as (1.1) in general do not admit classical solutions and the correct notion of weak solution is the one of viscosity solution ([2]). A theory of viscosity solutions for a general class of Hamilton-Jacobi equations with Caputo time-fractional derivative have been recently developed in [5, 17]. However, in these papers, the connection between Hamilton-Jacobi equations and the corresponding optimal control theory has not been pursued. In Proposition 3.2, we establish this connection for a.e. (sub-)solutions, but we are not able to show the corresponding property for viscosity solutions. Indeed, in the proof of the subsolution and supersolution conditions, applying the Ito's formula as in the classical viscosity 
solution argument, we get an equation involving Riemann-Liouville time-fractional derivative, see for example (3.9). The delicate point is that, for passing from (3.9) to (3.10), we perform a fractional integration and therefore we need that the equation is satisfied globally, while the notion of viscosity solution is only local. The connection with viscosity solutions, as defined in [5, 17], offers an interesting perspective for future research.

\section{Integral formula and numerical example}

We propose an example in order to show a comparison between $u$, the viscosity solution of the classical HamiltonJacobi equation (1.1), and $u_{\beta}$, the a.e. solution of the time-fractional Hamilton-Jacobi equation (1.6) given by (2.4). We start rewriting formula (2.4) as

$$
u_{\beta}(x, t)=\int_{0}^{\infty} \min _{y \in \mathbb{R}^{d}}\left\{r L\left(\frac{x-y}{r}\right)+g(y)\right\} \mathcal{E}_{\beta}(r, t) d r=\int_{0}^{\infty} u(x, r) \mathcal{E}_{\beta}(r, t) d r
$$

where $u$ is given by the formula $(1.4)$ and $\mathcal{E}_{\beta}(\cdot, t)$ is the PDF of $E_{t}$. Recalling (2.2), (4.1) can be also rewritten as

$$
u_{\beta}(x, t)=\frac{t}{\beta} \int_{0}^{\infty} u(x, s) \frac{\mathcal{D}_{\beta}\left(t s^{-1 / \beta}\right)}{s^{1+\frac{1}{\beta}}} d s .
$$

We will use formula (4.2) to compute the function $u_{\beta}$. We assume that the function $u$ is known in order to avoid additional numerical errors due to its approximation which could further affect $u_{\beta}$ and hide some important properties. Moreover we approximate the integral by a quadrature formula and we employ the Matlab toolbox Stable Distribution [12] to compute $\mathcal{D}_{\beta}(s)$. The toolbox requires 4 parameters $(\hat{\alpha}, \hat{\beta}, \hat{\gamma}, \hat{\delta})$ in order to compute a stable distribution (see [16]). For the distribution $\mathcal{D}_{\beta}$ corresponding to the value $\beta=0.4,0.5,0.6,0.8$ used in the test, we consider the following parameters

\begin{tabular}{c|c|c|c|c|} 
& $\hat{\alpha}$ & $\hat{\beta}$ & $\hat{\gamma}$ & $\hat{\delta}$ \\
\hline$\beta=0.4$ & $\beta$ & 1 & $\gamma_{c}$ & $\gamma_{c}-0.15$ \\
\hline$\beta=0.5$ & $\beta$ & 1 & $\gamma_{c}$ & $\gamma_{c}$ \\
\hline$\beta=0.6$ & $\beta$ & 1 & $\gamma_{c}$ & $\gamma_{c}+0.15$ \\
\hline$\beta=0.8$ & $\beta$ & 1 & $\gamma_{c}$ & $\gamma_{c}+0.5$ \\
\hline
\end{tabular}

having set $\gamma_{c}=\frac{1}{2}$. We briefly describe the algorithm:

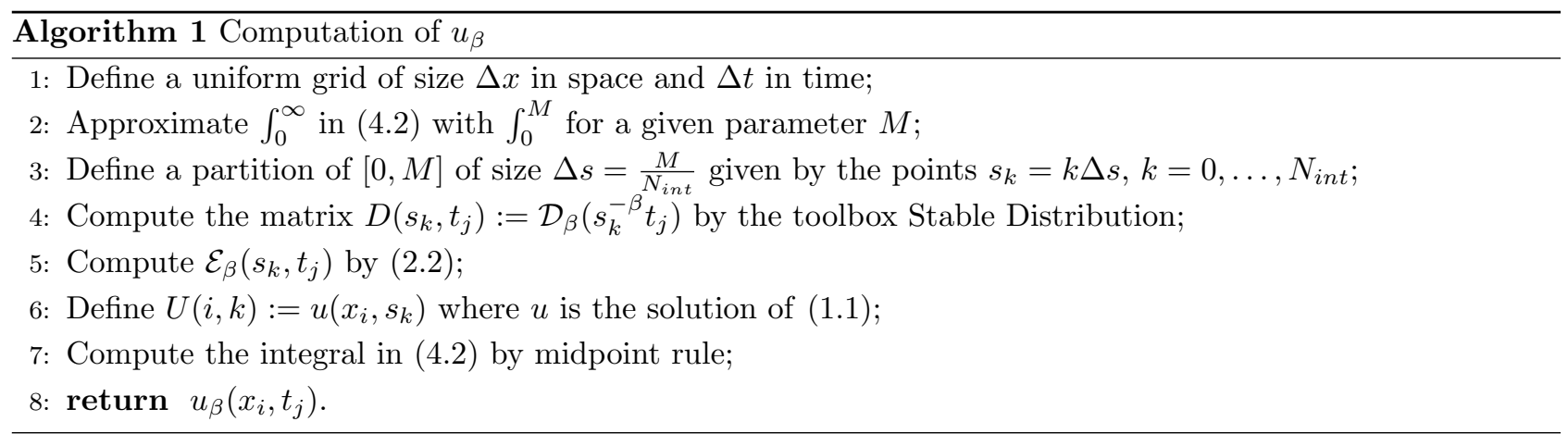

We consider

$$
\partial_{t} u+\frac{|D u|^{2}}{2}=0, \quad(x, t) \in \mathbb{R} \times(0, \infty),
$$


with the initial condition

$$
g(x)=\min \left\{0, x^{2}-1\right\} .
$$

In this case, the solution of (4.3) is given by

$$
u(x, t)=\min \left\{0, \frac{x^{2}}{1+2 t}-1\right\} .
$$

We compute $u_{\beta}$ by means of formula (4.2). Comparing the behavior of $u_{\beta}$ and $u$ in Figure 1 , we can see that for
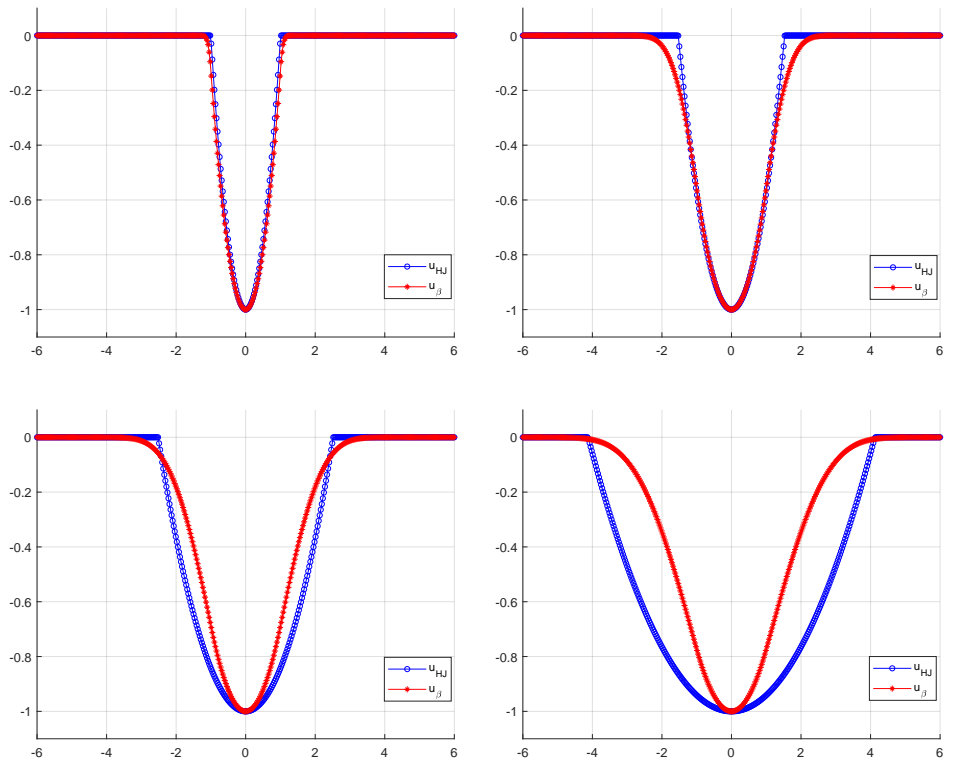

Figure 1: Comparison between $u$ and $u_{\beta}$ for $\beta=\frac{1}{2}$ at time $t=0.005$ (left-top), $t=0.5$ (right-top), $t=2$ (left-bottom), and $t=8$ (right-bottom).

small times the evolution of $u_{\beta}$ is faster than the one of $u$, since $u_{\beta}(x, t) \leq u(x, t)$ and $\operatorname{supp}(u(t)) \subset \operatorname{supp}\left(u_{\beta}(t)\right)$ for $t \leq 0.5$. While the time increases, the evolution of $u_{\beta}$ slows down with respect to the one of $u$. It is also interesting to observe the more regular behavior of $u_{\beta}$ in the space variable. Indeed the initial edge of $g$ is instantaneously smoothed for the fractional equation. We also observe that $u_{\beta}$ is not $C^{2}$ in space and a "memory" of the initial edge of $g$ is preserved in the second derivative.

\section{References}

[1] Allen, M.; Caffarelli, L.; Vasseur, A., A parabolic problem with a time-fractional derivative. Arch. Ration. Mech. Anal. 221 (2016), 603-630.

[2] Bardi, M., Capuzzo Dolcetta, I., Optimal Control and Viscosity Solutions of Hamilton-Jacobi-Bellman equations. Birkhäuser, Boston, 1997.

[3] Camilli, F.; De Maio, R., A time-fractional mean field game, Adv. Differential Equations, to appear. 

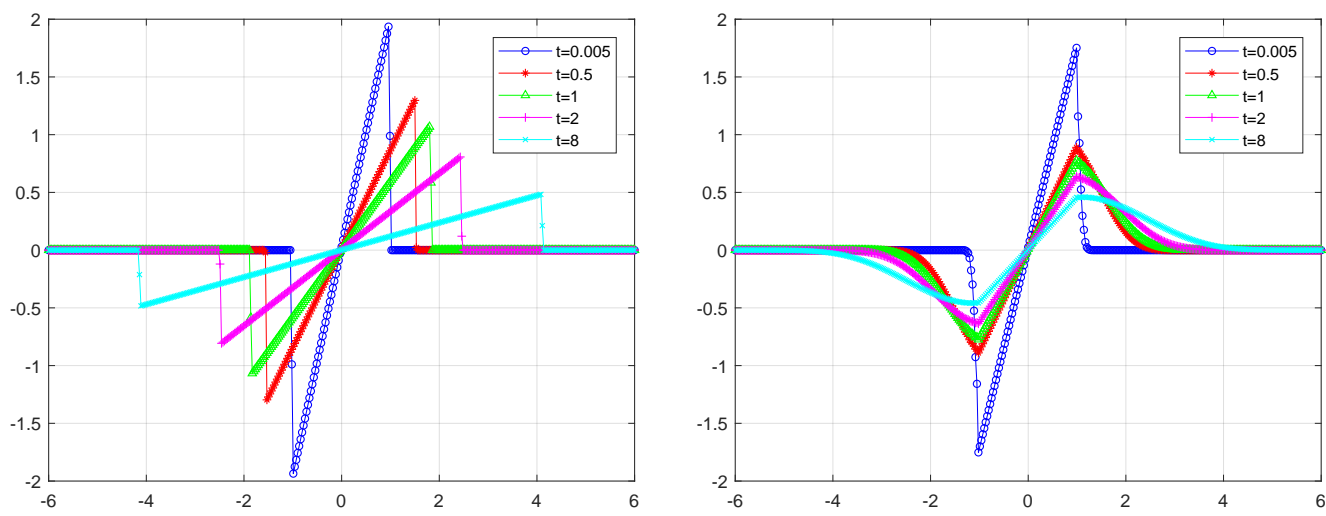

Figure 2: Space derivative of $u$ (left) and $u_{\beta}$ with $\beta=\frac{1}{2}$ (right), at different times.

[4] Evans, L. C., Partial Differential Equations, Grad. Stud. Math., vol. 19, Amer. Math. Soc., Providence, RI, 1998.

[5] Giga, Y.; Namba, T., Well-posedness of Hamilton-Jacobi equations with Caputo's time-fractional derivative, Comm. Partial Differential Equations 42 (2017), no. 7, 1088-1120.

[6] Kobayashi, K., Stochastic calculus for a time-changed semimartingale and the associated stochastic differential equations. J. Theor. Prob. 24 (2011), no. 3, 789-820.

[7] Li, L.; Liu, J., Some compactness criteria for weak solutions of time fractional pdes. SIAM Journal on Mathematical Analysis, 50 (2018), 3963-3995.

[8] Lions, P.L., Generalized solutions of Hamilton-Jacobi equations, Research Notes in Mathematics, Vol. 69, Pitman Advanced Publishing Program, Boston, 1982.

[9] Luchko, Y.; Yamamoto, M., General time-fractional diffusion equation: some uniqueness and existence results for the initial-boundary-value problems. Fract. Calc. Appl. Anal. 19 (2016), no. 3, 676-695.

[10] Magdziarz, M.; Gajda, J.; Zorawik, T., Comment on fractional Fokker-Planck equation with space and time dependent drift and diffusion, J. Stat. Phys. 154 (2014), no. 5, 1241-1250.

[11] Mainardi, F., Fractional Calculus and Waves in Linear Viscoelasticity, Imperial College Press, London, 2010.

[12] Mathworks, Stable Distribution toolbox, http://www.mathworks.com/help/stats/ stable-distribution.html.

[13] Meerschaert, M.; Scheffler, H.-P., Limit theorems for continuous-time random walks with infinite mean waiting times, J. Appl. Prob. 41 (2004), 623-638.

[14] Meerschaert, M.; Straka, P., Inverse Stable Subordinators, Math Model Nat Phenom. 8 (2013), no. 2, 1-16.

[15] Metzler, R.; Klafter, J., The random walk's guide to anomalous diffusion: A fractional dynamics approach, Phys. Rep. 339 (2000), no.1, 77 pp. 
[16] Penson, K. A.; Garska K., Exact and explicit probability densities for one-sided Lévy stable distributions, Phys. Rev. Lett. 105 (2010), no. 21, 2106-2110.

[17] Topp, E.; Yangari, M., Existence and uniqueness for parabolic problems with Caputo time derivative, J. Differential Equations 262 (2017), no. 12, 6018-6046. 